\title{
Studies on Tissue Protease
}

\section{Influence of Metal Salts upon Tryptase Activity of Rabbit Liver}

(Directed by Asst. Prof. M. Hayakawa)

By

\author{
Fumio Ito \\ (伊藤 交雄)
}

From Prof. Matunaga's Medical Clinic, Faculty of Medicine, Hirosaki University, Hirosaki

(Received for publication, April 10, 1954)

Activation by cysteine of catheptase activity is well known, and its activating mechanism has been discussed by many scholars without resulting in definite theory. In this connection influences of various metal salts upon catheptase activity has also been made. Krebs' ${ }^{1 \prime}$ reported that gelatin hydrolysis through catheptase of swine kidney extract has been inhibited by $\mathrm{Cu}, \mathrm{Ag}, \mathrm{Au}, \mathrm{Zn}, \mathrm{Cd}$, or by $\mathrm{Hg}$. According to Nakayama ${ }^{2 !}$ who studied catheptic kidney proteolysis, $\mathrm{CuSO}_{4}$ or $\mathrm{HgCl}_{2}$ was most strongly inhibitory. This was also the case when catheptic proteolysis of swine lung was studied by Onishi ${ }^{3}$, by whom it was shown that cysteine subdued the inhibitory action of $\mathrm{Ag}, \mathrm{Cu}$, or $\mathrm{Hg}$ but that no activation by $\mathrm{FeSO}_{4}$ or $\mathrm{FeCl}_{3}$ had occurred.

On the other hand Hayakawa ${ }^{4)}$ and $\mathrm{Ito}^{51}$ found that tryptase of human gastric mucosa and rabbit liver, contrary to catheptase, underwent no activation or seemed to be rather inhibited by cysteine. So influence of heavy metal salts upon tryptase is expected to differ from those upon catheptase.

\section{EXPERIMENTAL}

\section{Method}

The methods are the same as those previously described ${ }^{4}$.

The enzyme solution was prepared from liver, from which blood had been removed by means of washing through portal vein with aqua dest.

Reaction mixture: $20 \mathrm{cc}$. of substrate-buffer $(\mathrm{pH} 7.8)$ solution and $3 \mathrm{cc}$. of enzyme solution were mixed with $3 \mathrm{cc}$. of solution of various metal salts and then incubated under toluene at $37^{\circ} \mathrm{C}$ for digestion. Molar concentrations of salt in the digestion mixture are given in the Tables.

For controlling $3 \mathrm{cc}$. of metal salt solution is substituted with buffer 
solution of equal quantity. On the other hand, general control experiments without substrate were carried out with and without metal salt under same condition. Increased activity with respect to each $4 \mathrm{cc}$. of digestion mixture is formol-titrated with $N / 10-\mathrm{NaOH}$, and corrected values with general control values are tabulated.

\section{Results with Discussion}

$\mathrm{ZnSO}_{4}$ or $\mathrm{Fe}_{2}\left(\mathrm{SO}_{4}\right)_{3}$ appears, in concentration of $1 / 4,000 \mathrm{M}$, already somewhat to retard the hydrolysis amount after 120 hours' incubation of casein or gelatin, and $\mathrm{HgCl}_{2}$ or $\mathrm{CuSO}_{4}$ appears so in that of $1 / 2,000 \mathrm{M}$. (Table I). Inhibitory effects of these metal salts are first manifestly observed in concentration of over $1 / 500 M$. These inhibitory effects appear to become the more obvious when the times of incubation is the longer.

Inhibitory effect of $\mathrm{AgNO}_{3}, \mathrm{NiSO}_{4}$ or of $\mathrm{CoCl}_{2}$ seems less marked

\section{TABLE I}

Influence of $\mathrm{ZnSO}_{4}, \mathrm{Fe}_{2}\left(\mathrm{SO}_{4}\right)_{3}, \mathrm{HgCl}_{2}$ or $\mathrm{CuSO}_{4}$ upon Liver

Tryptase Activity of Rabbit at pH 7.8

Increases of acidity in $4 \mathrm{cc}$. digestion mixture : cc. of $0.1 \mathrm{~N} \mathrm{NaOH}$.

\begin{tabular}{|c|c|c|c|c|c|c|c|c|c|}
\hline \multirow{2}{*}{ Salt } & \multirow{2}{*}{ Substrate } & \multirow{2}{*}{$\begin{array}{l}\text { Time } \\
\text { in hrs. }\end{array}$} & \multicolumn{7}{|c|}{ Molar concentration of salts } \\
\hline & & & 0 & $1 / 8,000$ & $1 / 4,000$ & $1 / 2,000$ & $1 / 1,000$ & $1 / 500$ & 1,1000 \\
\hline \multirow{2}{*}{$\mathrm{ZnSO}_{4}$} & Casein & $\begin{array}{r}24 \\
72 \\
120\end{array}$ & $\begin{array}{l}0.15 \\
0.25 \\
0.4\end{array}$ & $\begin{array}{l}0.15 \\
0.25 \\
0.35\end{array}$ & $\begin{array}{l}0.1 \\
0.2 \\
0.25\end{array}$ & $\begin{array}{l}0.1 \\
0.2 \\
0.25\end{array}$ & $\begin{array}{l}0.1 \\
0.15 \\
0.2\end{array}$ & $\begin{array}{l}0.05 \\
0.1 \\
0.15\end{array}$ & $\begin{array}{l}0.05 \\
0.1 \\
0.15\end{array}$ \\
\hline & Gelatin & $\begin{array}{r}24 \\
72 \\
120\end{array}$ & $\begin{array}{l}0.15 \\
0.25 \\
0.3\end{array}$ & $\begin{array}{l}0.1 \\
0.15 \\
0.2\end{array}$ & $\begin{array}{l}0.1 \\
0.15 \\
0.2\end{array}$ & $\begin{array}{l}0.1 \\
0.15 \\
0.15\end{array}$ & $\begin{array}{l}0.1 \\
0.15 \\
0.15\end{array}$ & $\begin{array}{l}0.05 \\
0.1 \\
0.15\end{array}$ & $\begin{array}{l}0.05 \\
0.1 \\
0.15\end{array}$ \\
\hline \multirow{2}{*}{$\mathrm{Fe}_{2}\left(\mathrm{SO}_{4}\right)_{3}$} & Casein & $\begin{array}{r}24 \\
72 \\
120\end{array}$ & $\begin{array}{l}0.1 \\
0.25 \\
0.45\end{array}$ & $\begin{array}{l}0.1 \\
0.25 \\
0.45\end{array}$ & $\begin{array}{l}0.05 \\
0.25 \\
0.35\end{array}$ & $\begin{array}{l}0.05 \\
0.2 \\
0.35\end{array}$ & $\begin{array}{l}0.05 \\
0.15 \\
0.3\end{array}$ & $\begin{array}{l}0.05 \\
0.1 \\
0.25\end{array}$ & $\begin{array}{l}0.05 \\
0.1 \\
0.25\end{array}$ \\
\hline & Gelatin & $\begin{array}{r}24 \\
72 \\
120\end{array}$ & $\begin{array}{l}0.1 \\
0.2 \\
0.3\end{array}$ & $\begin{array}{l}0.05 \\
0.15 \\
0.25\end{array}$ & $\begin{array}{l}0.05 \\
0.15 \\
0.2\end{array}$ & $\begin{array}{l}0.05 \\
0.15 \\
0.2\end{array}$ & $\begin{array}{l}0.05 \\
0.1 \\
0.2\end{array}$ & $\begin{array}{l}0.05 \\
0.1 \\
0.15\end{array}$ & $\begin{array}{l}0.05 \\
0.1 \\
0.15\end{array}$ \\
\hline \multirow[t]{2}{*}{$\mathrm{HgCl}_{2}$} & Casein & $\begin{array}{r}24 \\
72 \\
120\end{array}$ & $\begin{array}{l}0.1 \\
0.25 \\
0.3\end{array}$ & $\begin{array}{l}0.1 \\
0.25 \\
0.3\end{array}$ & $\begin{array}{l}0.1 \\
0.25 \\
0.25\end{array}$ & $\begin{array}{l}0.1 \\
0.25 \\
0.2\end{array}$ & $\begin{array}{l}0.05 \\
0.1 \\
0.15\end{array}$ & $\begin{array}{l}0.05 \\
0.1 \\
0.15\end{array}$ & $\begin{array}{l}0.05 \\
0.1 \\
0.15\end{array}$ \\
\hline & Gelatin & $\begin{array}{r}24 \\
72 \\
120\end{array}$ & $\begin{array}{l}0.15 \\
0.15 \\
0.2\end{array}$ & $\begin{array}{l}0.15 \\
0.15 \\
0.2\end{array}$ & $\begin{array}{l}0.1 \\
0.1 \\
0.2\end{array}$ & $\begin{array}{l}0.05 \\
0.1 \\
0.2\end{array}$ & $\begin{array}{l}0.05 \\
0.05 \\
0.1\end{array}$ & $\begin{array}{l}0.05 \\
0.05 \\
0.1\end{array}$ & $\begin{array}{l}0.05 \\
0.05 \\
0.1\end{array}$ \\
\hline \multirow{2}{*}{$\mathrm{CuSO}_{4}$} & Casein & $\begin{array}{r}24 \\
72 \\
120\end{array}$ & $\begin{array}{l}0.1 \\
0.2 \\
0.3\end{array}$ & $\begin{array}{l}0.1 \\
0.2 \\
0.3\end{array}$ & $\begin{array}{l}0.1 \\
0.15 \\
0.3\end{array}$ & $\begin{array}{l}0.05 \\
0.1 \\
0.2\end{array}$ & $\begin{array}{l}0.05 \\
0.1 \\
0.1\end{array}$ & $\begin{array}{l}0.05 \\
0.1 \\
0.1\end{array}$ & $\begin{array}{l}0.05 \\
0.1 \\
0.1\end{array}$ \\
\hline & Gelatin & $\begin{array}{r}24 \\
72 \\
120\end{array}$ & $\begin{array}{l}0.05 \\
0.2 \\
0.25\end{array}$ & $\begin{array}{l}0.05 \\
0.2 \\
0.25\end{array}$ & $\begin{array}{l}0.05 \\
0.2 \\
0.25\end{array}$ & $\begin{array}{l}0.05 \\
0.1 \\
0.15\end{array}$ & $\begin{array}{l}0 \\
0.1 \\
0.15\end{array}$ & $\begin{array}{l}0 \\
0.05 \\
0.1\end{array}$ & $\begin{array}{l}0 \\
0.05 \\
0.1\end{array}$ \\
\hline
\end{tabular}


than above, because retardation of hydrolysis is observed only by 120 hrs.' digestion or in concentration of over $1 / 500 \mathrm{M}$. (Table II). III).

$\mathrm{FeSO}_{4}, \mathrm{~K}_{8} \mathrm{Fe}(\mathrm{CN})_{6}$ and $\mathrm{CdSO}_{4}$ neither inhibit nor activate (Table

\section{TABLE II}

Influence of $\mathrm{AgNO}_{3}, \mathrm{NiSO}_{4}$ or $\mathrm{CoCl}_{2}$ upon Tryptase

Activity of Rabbit Liver at pH 7.8

Increases of acidity in $4 \mathrm{cc}$. digestion mixture; cc. of $0.1 \mathrm{~N} \mathrm{NaOH}$.

\begin{tabular}{|c|c|c|c|c|c|c|c|c|c|}
\hline \multirow{2}{*}{ Salt } & \multirow{2}{*}{ Substrate } & \multirow{2}{*}{$\begin{array}{l}\text { Time } \\
\text { in } \\
\text { hrs. }\end{array}$} & \multicolumn{7}{|c|}{ Molar concentration of salts } \\
\hline & & & 0 & $1 / 8,000$ & $1 / 4,000$ & $1 / 2,000$ & $1 / 1,000$ & $1 / 500$ & $1 / 100$ \\
\hline \multirow{2}{*}{$\mathrm{AgNO}_{3}$} & Casein & $\begin{array}{r}24 \\
72 \\
120\end{array}$ & $\begin{array}{l}0.1 \\
0.25 \\
0.3\end{array}$ & $\begin{array}{l}0.1 \\
0.25 \\
0.25\end{array}$ & $\begin{array}{l}0.1 \\
0.2 \\
0.25\end{array}$ & $\begin{array}{l}0.1 \\
0.15 \\
0.15\end{array}$ & $\begin{array}{l}0.1 \\
0.15 \\
0.15\end{array}$ & $\begin{array}{l}0.1 \\
0.15 \\
0.15\end{array}$ & $\begin{array}{l}0.1 \\
0.1 \\
0.15\end{array}$ \\
\hline & Gelatin & $\begin{array}{r}24 \\
72 \\
120\end{array}$ & $\begin{array}{l}0.05 \\
0.15 \\
0.25\end{array}$ & $\begin{array}{l}0.1 \\
0.1 \\
0.25\end{array}$ & $\begin{array}{l}0.1 \\
0.1 \\
0.2\end{array}$ & $\begin{array}{l}0.05 \\
0.1 \\
0.2\end{array}$ & $\begin{array}{l}0.05 \\
0.1 \\
0.2\end{array}$ & $\begin{array}{l}0.05 \\
0.1 \\
0.2\end{array}$ & $\begin{array}{l}0.05 \\
0.05 \\
0.15\end{array}$ \\
\hline \multirow{2}{*}{$\mathrm{NiSO}_{4}$} & Casein & $\begin{array}{r}24 \\
72 \\
120\end{array}$ & $\begin{array}{l}0.1 \\
0.2 \\
0.3\end{array}$ & $\begin{array}{l}0.1 \\
0.2 \\
0.3\end{array}$ & - & $\begin{array}{l}0.1 \\
0.15 \\
0.25\end{array}$ & 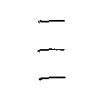 & $\begin{array}{l}0.05 \\
0.05 \\
0.2\end{array}$ & $\begin{array}{l}0.05 \\
0.05 \\
0.1\end{array}$ \\
\hline & Gelatin & $\begin{array}{r}24 \\
72 \\
120\end{array}$ & $\begin{array}{l}0.05 \\
0.15 \\
0.2\end{array}$ & $\begin{array}{l}0.05 \\
0.1 \\
0.1\end{array}$ & $\overline{-}$ & $\begin{array}{l}0.05 \\
0.1 \\
0.1\end{array}$ & I & $\begin{array}{l}0.05 \\
0.05 \\
0.05\end{array}$ & $\begin{array}{l}0.05 \\
0.05 \\
0.05\end{array}$ \\
\hline \multirow{2}{*}{$\mathrm{CoCl}_{2}$} & Casein & $\begin{array}{r}24 \\
72 \\
120\end{array}$ & $\begin{array}{l}0.1 \\
0.2 \\
0.3\end{array}$ & $\begin{array}{l}0.1 \\
0.2 \\
0.3\end{array}$ & $=$ & $\begin{array}{l}0.1 \\
0.15 \\
0.2\end{array}$ & $=$ & $\begin{array}{l}0.1 \\
0.1 \\
0.15\end{array}$ & $\begin{array}{l}0.05 \\
0.1 \\
0.15\end{array}$ \\
\hline & Gelatin & $\begin{array}{r}24 \\
72 \\
120\end{array}$ & $\begin{array}{l}0.05 \\
0.15 \\
0.25\end{array}$ & $\begin{array}{l}0.1 \\
0.15 \\
0.3\end{array}$ & E & $\begin{array}{l}0.05 \\
0.1 \\
0.15\end{array}$ & $\bar{E}$ & $\begin{array}{l}0.05 \\
0.05 \\
0.05\end{array}$ & $\begin{array}{l}0.05 \\
0.05 \\
0.05\end{array}$ \\
\hline
\end{tabular}

It is, however, noteworthy that $\mathrm{MnSO}_{4}$ seems, in concentration of over $1 / 2,000 \mathrm{M}$, by $72-120$ hours' digestion, to be accelerating for casein or gelatin hydrolysis (Table IV). The effect of $\mathrm{K}_{4} \mathrm{Fe}(\mathrm{CN})_{6}$ is, in concentration of over $1 / 2,000-1,000 M$, similarly accelerating (Table IV). But there is some apprehension that one might confuse enzymes, for which activity manganese sulfate is in fact activating, because enzyme solution used contains not only tryptase but also catheptase or ereptase, and manganese sulfate is known to activate dipeptidase activity. Avoiding such confusion an activating effect of manganese sulfate for tryptase is, however, evidenced because the same results are obtained from another experiment (Table V), for which enzyme solution, made by means of acid treatment ${ }^{\text {t) }}$, exclusive of catheptase, ereptase and dipeptidase (Table V-b) is employed.

Next manganese sulfate was examined for its effect in the presence of 
TABLE III

Influence of $\mathrm{FeSO}_{4}, \mathrm{~K}_{3} \mathrm{Fe}(\mathrm{CN})_{6}$ or $\mathrm{CdSO}_{4}$ upon Tryptase

Activity of Rabbit Liver at $\mathrm{pH} 7.8$

Increases of acidity in $4 \mathrm{cc}$. digestion mixture; cc. of $0.1 \mathrm{~N} \mathrm{NaOH}$.

\begin{tabular}{|c|c|c|c|c|c|c|c|}
\hline \multirow{2}{*}{ Salt } & \multirow{2}{*}{ Substrate } & \multirow{2}{*}{$\begin{array}{l}\text { Time } \\
\text { in } \\
\text { hrs. }\end{array}$} & \multicolumn{5}{|c|}{ Molar concentration of salts } \\
\hline & & & 0 & $1 / 5,000$ & $1 / 1,000$ & $1 / 500$ & $1 / 100$ \\
\hline \multirow{2}{*}{$\mathrm{FeSO}_{4}$} & Cásein & $\begin{array}{r}24 \\
72 \\
120\end{array}$ & $\begin{array}{l}0.05 \\
0.15 \\
0.2\end{array}$ & $\begin{array}{l}0.1 \\
0.15 \\
0.2\end{array}$ & $\begin{array}{l}0.1 \\
0.2 \\
0.25\end{array}$ & $\begin{array}{l}0.1 \\
0.2 \\
0.25\end{array}$ & $\begin{array}{l}0.1 \\
0.2 \\
0.2\end{array}$ \\
\hline & Gelatin & $\begin{array}{r}24 \\
72 \\
120\end{array}$ & $\begin{array}{l}0.05 \\
0.1 \\
0.15\end{array}$ & $\begin{array}{l}0.05 \\
0.05 \\
0.1\end{array}$ & $\begin{array}{l}0.05 \\
0.05 \\
0.1\end{array}$ & $\begin{array}{l}0.05 \\
0.05 \\
0.1\end{array}$ & $\begin{array}{l}0.05 \\
0.05 \\
0.2\end{array}$ \\
\hline \multirow{2}{*}{$\mathrm{K}_{3} \mathrm{Fe}(\mathrm{CN})_{6}$} & Casein & $\begin{array}{r}24 \\
72 \\
120\end{array}$ & $\begin{array}{l}0.15 \\
0.25 \\
0.35\end{array}$ & $\begin{array}{l}0.15 \\
0.25 \\
0.35\end{array}$ & $\begin{array}{l}0.15 \\
0.25 \\
0.35\end{array}$ & $\begin{array}{l}0.15 \\
0.25 \\
0.35\end{array}$ & $\begin{array}{l}0.15 \\
0.25 \\
0.35\end{array}$ \\
\hline & Gelatin & $\begin{array}{r}24 \\
72 \\
120\end{array}$ & $\begin{array}{l}0.05 \\
0.15 \\
0.2\end{array}$ & $\begin{array}{l}0.05 \\
0.15 \\
0.15\end{array}$ & $\begin{array}{l}0.05 \\
0.15 \\
0.15\end{array}$ & $\begin{array}{l}0.1 \\
0.15 \\
0.15\end{array}$ & $\begin{array}{l}0.1 \\
0.15 \\
0.15\end{array}$ \\
\hline \multirow{2}{*}{$\mathrm{CdSO}_{4}$} & Casein & $\begin{array}{r}24 \\
72 \\
120\end{array}$ & $\begin{array}{l}0.1 \\
0.15 \\
0.25\end{array}$ & $\begin{array}{l}0.1 \\
0.15 \\
0.2\end{array}$ & $\begin{array}{l}0.1 \\
0.15 \\
0.2\end{array}$ & $\begin{array}{l}0.1 \\
0.1 \\
0.15\end{array}$ & $\begin{array}{l}0.05 \\
0.15 \\
0.2\end{array}$ \\
\hline & Gelatin & $\begin{array}{r}24 \\
72 \\
120\end{array}$ & $\begin{array}{l}0.1 \\
0.15 \\
0.25\end{array}$ & $\begin{array}{l}0.05 \\
0.1 \\
0.2\end{array}$ & $\begin{array}{l}0.05 \\
0.05 \\
0.2\end{array}$ & $\begin{array}{l}0.05 \\
0.05 \\
0.2\end{array}$ & $\begin{array}{l}0.05 \\
0.05 \\
0.2\end{array}$ \\
\hline
\end{tabular}

dialyzed albumin solution, and activating effect observed was duplicated, i.e. the hydrolysis amount became larger than when each of these two activators alone was present (Table VI). But manganese sulfate appears to be somewhat inferior to dialyzed albumin solution in activating effect. From this the suggestion can be made that manganese sulfate may have its own mechanism for activation other than that of dialyzed albumin solution.

Mode of influences of heavy metal salts upon tryptase activity seems, in. comparison with those upon catheptase, characteristic in the following respects: Their influences upon tryptase, either activating or inhibiting, appear to be rather delayed, because their influences become more obvious as the time of digestion is increased. Retardations of catheptic hydrolysis are, during the lapse of the time of digestion, more or less restored ${ }^{2-3)}$. And the metal salt which is strongly inhibitory against catheptic hydrolysis appears to be not always so against tryptic hydrolysis. Therefore these disimilarities together with the effects of cysteine, suggest that the mechanism of each of these enzyme actions may differ.

On the other hand tryptase in rabbit liver can be definitely said to exist and it manifests itself highly after duplicate activation through dialyzed 
albumin solution and manganese sulfate together.

\section{SUMMARY}

Influences of metal salts upon liver tryptase activity were investigated with following results :

1. $\mathrm{ZnSO}_{4}, \mathrm{Fe}_{2} \mathrm{SO}_{4}, \mathrm{HgCl}_{2}$ or $\mathrm{CuSO}_{4}$ is inhibitory to casein or gelatin hydrolysis through rabbit liver tryptase by $72-120$ hours' digestion in concentration of over $1 / 2000 M$.

2. The inhibitory effect of $\mathrm{AgNO}_{3}, \mathrm{NiSO}_{4}$ or $\mathrm{CoCl}_{2}$ is less than that of the above, and $\mathrm{FeSO}_{4}, \mathrm{~K}_{3} \mathrm{Fe}(\mathrm{CN})_{6}$ and $\mathrm{CdSO}_{4}$ neither inhibit nor activate.

\section{TABLE IV}

Influence of $\mathrm{MnSO}_{4}$ or $\mathrm{K}_{4} \mathrm{Fe}(\mathrm{GN})_{6}$ upon Tryptase

Activity of Rabbit at $\mathrm{pH} 7.8$

Increases of acidity in $4 \mathrm{cc}$. digestion mixture are expressed in cc.

of $0.1 \mathrm{~N} \mathrm{NaOH}$

\begin{tabular}{|c|c|c|c|c|c|c|c|c|c|}
\hline \multirow{2}{*}{ Salt } & \multirow{2}{*}{ Substrate } & \multirow{2}{*}{$\begin{array}{l}\text { Time } \\
\text { in } \\
\text { hrs. }\end{array}$} & \multicolumn{7}{|c|}{ Molar concentration of salts } \\
\hline & & & 0 & $1 / 8,000$ & $1 / 4,000$ & $1 / 2,000$ & $1 / 1,000$ & $1 / 500$ & $1 / 100$ \\
\hline \multirow{2}{*}{$\mathrm{MnSO}_{4}$} & Casein & $\begin{array}{r}24 \\
72 \\
120\end{array}$ & $\begin{array}{l}0.1 \\
0.2 \\
0.3\end{array}$ & $\begin{array}{l}0.1 \\
0.25 \\
0.3\end{array}$ & $\begin{array}{l}0.1 \\
0.25 \\
0.35\end{array}$ & $\begin{array}{l}0.1 \\
0.3 \\
0.4\end{array}$ & $\begin{array}{l}0.15 \\
0.3 \\
0.4\end{array}$ & $\begin{array}{l}0.15 \\
0.35 \\
0.45\end{array}$ & $\begin{array}{l}0.15 \\
0.35 \\
0.45\end{array}$ \\
\hline & Gelatin & $\begin{array}{r}24 \\
72 \\
120\end{array}$ & $\begin{array}{l}0.1 \\
0.15 \\
0.15\end{array}$ & $\begin{array}{l}0.15 \\
0.15 \\
0.2\end{array}$ & $\begin{array}{l}0.1 \\
0.15 \\
0.2\end{array}$ & $\begin{array}{l}0.1 \\
0.2 \\
0.25\end{array}$ & $\begin{array}{l}0.1 \\
0.2 \\
0.25\end{array}$ & $\begin{array}{l}0.1 \\
0.25 \\
0.3\end{array}$ & $\begin{array}{l}0.1 \\
0.25 \\
0.3\end{array}$ \\
\hline \multirow{2}{*}{$\mathrm{K}_{4} \mathrm{Fe}(\mathrm{CN})_{6}$} & Casein & $\begin{array}{r}24 \\
72 \\
120\end{array}$ & $\begin{array}{l}0.2 \\
0.25 \\
0.3\end{array}$ & $\begin{array}{l}0.2 \\
0.25 \\
0.3\end{array}$ & $\begin{array}{l}0.2 \\
0.3 \\
0.3\end{array}$ & $\begin{array}{l}0.15 \\
0.35 \\
0.4\end{array}$ & $\begin{array}{l}0.15 \\
0.35 \\
0.35\end{array}$ & $\begin{array}{l}0.2 \\
0.35 \\
0.4\end{array}$ & $\begin{array}{l}0.15 \\
0.35 \\
0.45\end{array}$ \\
\hline & Gelatin & $\begin{array}{r}24 \\
72 \\
120\end{array}$ & $\begin{array}{l}0.1 \\
0.2 \\
0.2\end{array}$ & $\begin{array}{l}0.1 \\
0.2 \\
0.25\end{array}$ & $\begin{array}{l}0.1 \\
0.25 \\
0.25\end{array}$ & $\begin{array}{l}0.1 \\
0.25 \\
0.25\end{array}$ & $\begin{array}{l}0.1 \\
0.2 \\
0.3\end{array}$ & $\begin{array}{l}0.1 \\
0.25 \\
0.3\end{array}$ & $\begin{array}{l}0.1 \\
0.25 \\
0.3\end{array}$ \\
\hline
\end{tabular}

TABLE V

a. Influence of $\mathrm{MnSO}_{4}$ upon Enzyme Solution free from

Ereptase and Catheptase Activities

Increased acidity in $4 \mathrm{cc}$. digestion mixture; cc. of $0.1 \mathrm{~N} \mathrm{NaOH}$.

5 cc. enzyme solution was used. $\mathrm{pH}$ of digestion, 7.8 Substrate, casein

\begin{tabular}{c|c|c|c|c|c}
\hline \multirow{3}{*}{ Time in hrs. } & \multicolumn{5}{|c}{ Molar concentration of $\mathrm{MnSO}_{4}$} \\
\cline { 2 - 5 } & 0 & $1 / 10,000$ & $1 / 5,000$ & $1 / 1,000$ & $1 / 100$ \\
\hline 24 & 0.15 & 0.15 & 0.15 & 0.2 & 0.2 \\
72 & 0.25 & 0.3 & 0.3 & 0.4 & 0.4 \\
120 & 0.35 & 0.35 & 0.45 & 0.5 & 0.6
\end{tabular}


b. Proteolytic Activity at $\mathrm{pH} 7.8$ of Tissue Homogenate of Rabbit Liver, exposed to $\mathrm{pH} 3.5$ at $37^{\circ} \mathrm{C}$ for 40 minutes Increases of acidity in $4 \mathrm{cc}$. digestion mixture; cc. of $0.1 \mathrm{~N} \mathrm{NaOH}$.

\begin{tabular}{l|c|c|c}
\hline \multirow{2}{*}{ Substrate } & $\begin{array}{c}\text { Time in } \\
\text { hrs. }\end{array}$ & \multicolumn{2}{|c}{$\mathrm{pH}$ of digestion } \\
& 24 & 7.8 & 4.5 \\
\hline \multirow{4}{*}{ Casein } & 72 & 0.15 & 0 \\
& 120 & 0.25 & 0.05 \\
Gelatin & 0.35 & 0.05 \\
& 24 & 0.1 & 0 \\
Pepton & 0.2 & 0 \\
& 12 & 0.25 & 0 \\
M/20-Diglycin & 24 & 0 & 0 \\
& 72 & 0 & 0 \\
& 120 & 0 & 0 \\
& 24 & 0 & 0 \\
\end{tabular}

\section{TABLE VI}

Activation of Rabbit Liver Tryptase with combined Use of dialyzed Albumin solution and Maganese sulfate solution

$10 \mathrm{cc} .2 \%$-casein-buffer solution $+10 \mathrm{cc}$. dialyzed ov/serum albumin solution or buffer solution $+3 \mathrm{cc}$. enzyme solution $+3 \mathrm{cc}$. $\mathrm{MnSO}_{2}$ or buffer solution. Increases of acidity in $4 \mathrm{cc}$. digestion mixture; cc. of $0.1 \mathrm{~N} \mathrm{NaOH}$, $\mathrm{pH}$ of digestion 7.8 .

\begin{tabular}{c|c|c|c|c|c|c}
\hline $\begin{array}{c}\text { Time } \\
\text { in } \\
\text { hrs. }\end{array}$ & $\begin{array}{c}\text { without } \\
\text { albumin } \\
\text { and } \\
\mathrm{MnSO}_{4}\end{array}$ & $\begin{array}{c}\text { with } \\
\text { dialyzed } \\
\text { serum } \\
\text { albumin }\end{array}$ & $\begin{array}{c}\text { with } \\
\text { dialyzed } \\
\text { ovalbumin }\end{array}$ & $\begin{array}{c}\text { with } \\
M / 100- \\
\mathrm{MnSO}_{4}\end{array}$ & $\begin{array}{c}\text { with dialyzed } \\
\text { serum albumin } \\
\text { and } \mathrm{MnSO}_{4}\end{array}$ & $\begin{array}{c}\text { with dialyzed } \\
\text { ovalbumin } \\
\text { and } \mathrm{MnSO}_{4}\end{array}$ \\
\hline 24 & $0.15 ; 0.1$ & 0.4 & 0.45 & 0.35 & 0.6 & $0.6 ; 0.5$ \\
72 & $0.25 ; 0.3$ & 0.65 & 0.75 & 0.5 & 1.05 & $0.75 ; 0.75$ \\
120 & $0.35 ; 0.4$ & 0.9 & 0.9 & 0.7 & 1.35 & $1.5 ; 1.4$
\end{tabular}

3. $\mathrm{MnSO}_{4}$ or $\mathrm{K}_{4} \mathrm{Fe}(\mathrm{CN})_{6}$ activates casein hydrolysis by 72 hours' digestion in concentration of over $1 / 1000 M$. Such effect is recognizable even when catheptase and ereptase are removed by means of acid treatment $^{6)}$, and manganese sulfate is still activating for tryptase activity which is activated simultaneously in the presence of dialyzed albumin solution. In such a way tryptase is, in its highly active state, undoubtedly recognizable. on rabbit liver.

This work was carried out by a grant for development of scientific researches, given from the Ministry of Education. M. Hayakawa 


\section{References}

1) Krebs, Biochem. Z., 1930, 220, 289 ; ibid., 1931, 238, 174.

2) Nakayama, Tohoku J. Exp. Med., 1940, 38, 485.

3) Onishi, ibid., 1942, 43, 13.

4) Hayakawa, ibid., 1951, 53, 251.

5) Ito, ibid., 1954, 60, 367.

6) Utzino \& Sakai, J. Biochem., 1941, 33, 457. 